\title{
Two-Dimensional Product Warranty Cost Model under Preventive Maintenance Time Constraints
}

\author{
Qian Wang ${ }^{\mathrm{a}, \mathrm{b}}$, Zhonghua Cheng ${ }^{\mathrm{a},{ }^{*}}$, Zhiyong $\mathrm{Li}^{\mathrm{c}}$, and Yongsheng Bai ${ }^{\mathrm{a}}$ \\ ${ }^{a}$ Department of Equipment Command and Administration, Shijiazhuang Campus of Army Engineering University, Shijiazhuang, 050000, China \\ ${ }^{b}$ Ninth Comprehensive Training Base of Army, Zhangjiakou, 075000, China \\ ${ }^{c}$ Teaching and Researching Department, Shijiazhuang Campus of Army Engineering University, Shijiazhuang, 050000, China
}

\begin{abstract}
Providing high-quality product warranty service is an important part of the current competition among manufacturers for customers. On the basis of current warranty service, providing reasonable warranty service to customers more closely and practically is a problem that manufacturers must consider. On the basis of existing literature and considering the actual situation of manufacturers and customers, the time constraints of preventive maintenance in warranty service are proposed for the first time. Under the incomplete preventive maintenance strategy, the time of preventive maintenance during the warranty period is limited, and a two-dimensional product maintenance cost model under the time constraint is established to determine how to carry out preventive maintenance to obtain the ideal maintenance cost. The validity of the model is verified by an example, providing a basis for product two-dimensional warranty service decision-making.
\end{abstract}

Keywords: Time constraint; two-dimensional warranty; cost

(Submitted on December 13, 2018; Revised on January 14, 2019; Accepted on February 17, 2019)

(C) 2019 Totem Publisher, Inc. All rights reserved.

\section{Introduction}

At present, in the fierce market competition, in addition to having excellent performance, reasonable price, and excellent quality, after-sale warranty service plays an increasingly important role in product marketing. During the warranty period, good after-sale service has become an important factor in competition among manufacturers. According to the deadline of warranty service, the warranty service can be divided into one-dimensional warranty, two-dimensional warranty, and multidimensional warranty strategy [1]. At present, the two-dimensional warranty strategy has been widely used in many military and civil equipment maintenance services. Two-dimensional warranty covers a rectangular two-dimensional warranty area. Usually, the horizontal axis is used to indicate the use time and the vertical axis is used to indicate the use degree. The warranty service ends regardless of the time or degree of use arrived first. As shown in Figure 1, many military and civilian general vehicles are guaranteed by using time and driving mileage.

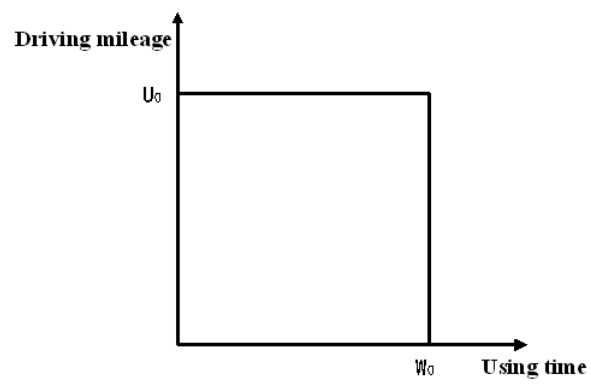

Figure 1. Two-dimensional warranty area

\footnotetext{
* Corresponding author.

E-mail address: bwrs69@sohu.com
} 
In the current warranty service, the maintenance cost during the warranty period is usually borne by the manufacturer, that is, after the product breaks down, the manufacturer carries out free maintenance. To reduce the cost of warranty and improve availability, preventive maintenance is necessary, because the cost and time of maintenance after product failure is usually greater than before [2-3]. At present, in some fields, the warranty service has gradually changed from a repairoriented maintenance strategy to a preventive maintenance strategy. Many scholars have proven the superiority of this strategy through research. Preventive maintenance during the warranty period can be divided into two categories: periodic maintenance and non-periodic preventive maintenance.

Huang established a cost model under periodic preventive maintenance strategy and obtained a maintenance scheme that maximizes the manufacturer's profit [4]. Wu studied the optimization of periodic preventive maintenance considering aging loss during the warranty period [5]. Huang analyzed the two-dimensional warranty cost under periodic preventive maintenance strategy [6]. Tao studied the influence of the number of preventive maintenance in the basic warranty period on the length of extended warranty period [7]. Alqahtani introduced preventive maintenance into remanufactured products. When the remaining life of the product reached a predetermined value, periodic preventive maintenance was carried out. The corresponding model was established, and the validity of the model was proven by simulation [8]. Liao considered carrying out periodic preventive maintenance within the warranty period and performing complete maintenance, incomplete maintenance, or predictive maintenance with different probabilities. Major repair was performed after failure. Finally, the preventive maintenance cycle with the lowest cost during the warranty period was determined [9]. Wang studied that periodic preventive maintenance should be carried out during the warranty period. Users should choose whether to carry out preventive maintenance or not, and two cost models should be established to optimize the user's income and manufacturer's warranty cost. The applicability of the model was verified by an example [10]. Wang investigated upgrading and preventive maintenance of second-hand products during the extended warranty period. It was divided into four schemes to study, and the applicability of different schemes was compared by examples [11]. Shafiee proposed preventive maintenance for products that deteriorate over time during the warranty period and assumed that the cost of each preventive maintenance was different. Considering from the manufacturer's point of view, the cost model within the warranty period was established [12]. Zhou divided the warranty period into different stages. The period of PM in each stage was different, which could solve the problem of higher maintenance cost in the later period of the warranty due to the decrease of failure rate [13]. Wang considered it from the manufacturer's point of view and built a joint model of basic warranty and extended warranty [14]. In this model, periodic incomplete preventive maintenance was carried out to minimize the maintenance cost during the warranty period. Park considered it from the manufacturer's point of view and carried out periodic preventive maintenance during the warranty period. Minimum maintenance or replacement maintenance depended on whether the repair time exceeded the threshold. Finally, the optimal value of preventive maintenance interval was determined to minimize the cost during the warranty period [15]. Nasrum studied the two-dimensional warranty contract of dump trucks and carried out periodic preventive maintenance in the two-dimensional warranty. In this paper, they provided two warranty strategies and proved that the second strategy was better than the first one. The method of optimum preventive maintenance cycle was given [16]. Cheng established a model of periodic preventive maintenance under the condition of delayed initial time, assuming that the cost of each preventive maintenance was different for products degraded with time, and proved the applicability and validity of the model [17]. Han took the cost in the warranty period as the objective and took the preventive maintenance interval in the two-dimensional warranty period as the variable to establish the model. It was also assumed that if there was a product failure during the preventive maintenance interval, it would be replaced directly; otherwise, it would be replaced when preventive maintenance occurs. Finally, the best preventive maintenance interval was determined [18].

There have also been many works focused on non-periodic preventive maintenance during the warranty period. Husniah divided the two-dimensional warranty period into five stages, and preventive maintenance was carried out only in two stages [19]. Huang established an aperiodic preventive maintenance model based on the reliability threshold. The time interval for incomplete preventive maintenance was optimized with the goal of maximizing the profit of the manufacturer. Furthermore, the effects of warranty period elasticity and price elasticity on the manufacturer's earnings were considered [20]. Lin formulated a non-periodic preventive maintenance strategy with reliability as the constraint. Through sensitivity analysis, it was proven that the model with high reliability constraints could not only improve the availability during the trial period, but also prolong the service life. However, its maintenance costs were higher [21].

In the current research on preventive maintenance strategy, models are built under the most ideal assumptions, without considering the time constraints of executive maintenance. Such theoretical research does not match the actual situation in engineering applications. Through consulting a large number of literatures related to the study of warranty service, no relevant research with time constraints has been found. This paper is the first to propose a decision-making model of maintenance service considering preventive maintenance under time constraints. The proposed time constraint mainly refers to the time limit for preventive maintenance, which mainly comes from users, manufacturers, and the natural environment. 
Only in a certain period of time can preventive maintenance be carried out. The new model can solve the practical problems existing in the current warranty service to a certain extent. It can be used for reference to reduce the cost of large-scale equipment warranty periods. The most important thing is to reduce the price of products and enhance the attractiveness of customers. It is very beneficial to both manufacturers and users. For large equipment, achieving complete maintenance is not practical, so preventive maintenance is usually incomplete maintenance. Therefore, this paper studies the decisionmaking model of equipment maintenance service under incomplete the preventive maintenance strategy.

The structure of the other sections is as follows. Section 2 introduces the basic situation of incomplete maintenance. Section 3 gives the cost model under time constraints. In order to verify the validity of the model, an example is given in Section 4. In Section 5, the results are discussed. The last section gives a summary and development direction.

\section{Incomplete Preventive Maintenance}

Incomplete maintenance can be divided into incomplete repairing maintenance and incomplete preventive maintenance. In this paper, we mainly discuss incomplete preventive maintenance. Incomplete preventive maintenance can restore the malfunctioning product. However, due to the limitations of maintenance equipment, maintenance capacity, and maintenance personnel, the product performance status will not be restored as new. Preventive maintenance of large equipment is usually incomplete maintenance. There are usually two ways to describe the effect of incomplete maintenance. One is to directly reduce the product failure rate, and the other is to reduce the failure rate by reducing the virtual time of product use. This article uses the second effect. In order to describe the change of product failure rate before and after incomplete preventive maintenance, a repair factor $\delta$ is introduced, where $(0<\delta<1)$. Assuming preventive maintenance at $T$, the performance of the product is improved and the failure rate is reduced to the same as $\delta T$. From the recurrence relation, the failure rate of the product can be obtained as follows:

$$
\begin{array}{lc}
\lambda_{0}(t)=\lambda(t), & 0<t<T_{1} \\
\lambda_{1}(t)=\lambda\left(t-\delta T_{1}\right), & T_{1}<t<T_{2} \\
\lambda_{2}(t)=\lambda\left(t-\delta T_{2}\right), & T_{2}<t<T_{3} \\
\ldots & \\
\lambda_{i}(t)=\lambda\left(t-\delta T_{i}\right), & T_{i}<t<T_{i+1}
\end{array}
$$

$\lambda_{i}(t)$ is the failure rate of the product after the $i^{\text {th }}$ incomplete preventive maintenance. $T_{i}$ is the $i^{\text {th }}$ preventive maintenance time. The effect of incomplete preventive maintenance on the product failure rate is shown in Figure 2.

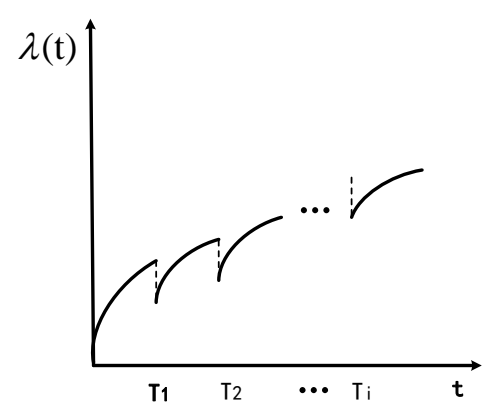

Figure 2. The impact of incomplete preventive maintenance on failure rate

\section{Two-Dimensional Warranty Cost Model for Products with Time Constraints}

\subsection{Model Hypothesis}

(1) After preventive maintenance, the failure rate of the product is between the repair as new and the repair as old, and the failure rate of the product does not change after the minimum maintenance. (For such a product with many components, replacing one component after failure will not affect the failure rate of the product.)

(2) During the two-dimensional warranty period, under the condition that the preventive maintenance time is limited, the manufacturer carries out preventive maintenance on the products. 
(3) The product has aging characteristics, and the failure rate increases with the increase of service time and degree.

(4) The degree of incomplete preventive maintenance is the same at each time.

(5) The maintenance time is much shorter than the warranty time. When establishing a cost model, the maintenance time can be neglected.

(6) Preventive maintenance is performed only during each time constraint.

\subsection{Establishment of Two-Dimensional Warranty Cost Model}

In the two-dimensional warranty period, the maintenance cost model for preventive maintenance with $n$ time constraints is established. The time limit and usage limit of the two-dimensional warranty period are $W_{0}$ and $U_{0}$, respectively. The warranty period ends when the product is used longer than $W_{0}$ or more than $U_{0}$. Assuming that the usage rate of a single product remains unchanged during the warranty period and different users have different usage rates, for batch products, the usage rate is a random variable. Assume that the manufacturer understands the distribution through previous product usage information and surveys, and the distribution function and probability density function of usage are $G(r)$ and $g(r)$, respectively. In the existing research, there are three main ways to express the two-dimensional failure rate, namely, the two-factor variable method, the composite scale method, and the usage rate method. Among them, the usage rate method is widely used. Assuming that the usage of product $u$ is a linear function of the usage time $t$, for a given usage rate $r$, there is $u=r \times t$. The failure rate function is $\lambda(t \mid r)$ when the product usage rate is $r$. The expression is [22] as follows: $\lambda(t \mid r)=\theta_{0}+\theta_{1} r+\theta_{2} t^{2}+\theta_{3} r t^{2}$. According to the climatic conditions in the area where the product is used, and through the agreement between the manufacturer and the user, it is determined that only during the $n$ intervals, $\left[T_{a 1}, T_{b 1}\right],\left[T_{a 2}, T_{b 2}\right], \cdots$, $\left[T_{a n}, T_{b n}\right]$, can the manufacturer carry out $n$ preventive maintenances for the products. The degree of preventive maintenance is incomplete maintenance, and the repair factor is $\delta$. The cost of each preventive maintenance is $C_{p}$. After the product breaks down, only the smallest maintenance is carried out, and the cost is $C_{f}$. Under this assumption, during the interval of preventive maintenance, the equipment failure obeys the non-homogeneous Poisson process (NHPP). Assume that $n$ preventive maintenance times are $T_{1}, T_{2}, \cdots, T_{n}, \cdots$, respectively, and $T_{1} \in\left[T_{a 1}, T_{b 1}\right], T_{2} \in\left[T_{a 2}, T_{b 2}\right], \cdots, T_{n} \in\left[T_{a n}, T_{b n}\right]$. That is to say, only one preventive maintenance can be done in each shadow area, as shown in Figure 3.

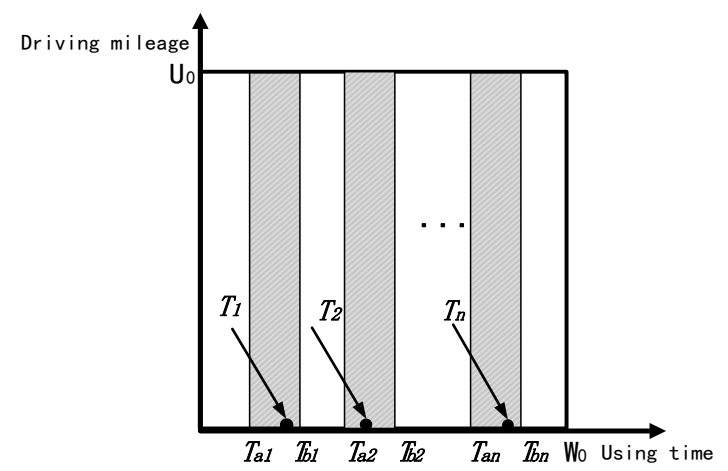

Figure 3. Preventive maintenance under time constraints

Due to the different utilization usage rate of equipment, the cost of $n$ preventive maintenances should be divided into $n+2$ cases. There are four cost expressions $\left(T_{0}=0\right)$.

(1) When $r \geq \frac{U_{0}}{T_{1}}$, no preventive maintenance is carried out, and the warranty service is terminated at the end of the driving mileage. It is known that in two consecutive preventive maintenance periods, the product failure follows a nonhomogeneous Poisson process, and the expected number of failure times is the integral of the failure rate in these two periods. The cost can be expressed as: 


$$
E_{1}(C)=C_{f} \int_{0}^{\frac{U_{0}}{r}} \lambda(t) \mathrm{d} t
$$

(2) When $\frac{U_{0}}{T_{m}} \leq r<\frac{U_{0}}{T_{m-1}},((m-1) \in[2, n]$, and $m$ is an integer). (m-1) times of preventive maintenance are carried out, and the warranty service is terminated at the end of the driving mileage. The cost is as follows:

$$
\begin{aligned}
E_{m}(C)=C_{f} \int_{0}^{T_{1}} \lambda(t) \mathrm{d} t+C_{f} \int_{T_{1}}^{T_{2}} \lambda\left(t-\delta T_{1}\right) \mathrm{d} t+\cdots+C_{f} \int_{T_{m-2}}^{T_{m-1}} \lambda\left(t-\delta T_{m-2}\right) \mathrm{d} t \\
+\cdots+C_{f} \int_{T_{m-1}}^{\frac{U_{0}}{r}} \lambda\left(t-\delta T_{m-1}\right) \mathrm{d} t+(m-1) C_{p} \\
=C_{f} \sum_{i=1}^{m-1} \int_{T_{i-1}}^{T_{i}} \lambda\left(t-\delta T_{i-1}\right) \mathrm{d} t+C_{f} \int_{T_{m-1}}^{\frac{U_{0}}{r}} \lambda\left(t-\delta T_{m-1}\right) \mathrm{d} t+(m-1) C_{p}
\end{aligned}
$$

(3) When $\frac{U_{0}}{W_{0}} \leq r<\frac{U_{0}}{T_{n}}, n$ times of preventive maintenance are carried out and the warranty service is terminated at the end of the driving mileage. The cost is as follows:

$$
\begin{aligned}
E_{n+1}(C)= & C_{f} \int_{0}^{T_{1}} \lambda(t) \mathrm{d} t+C_{f} \int_{T_{1}}^{T_{2}} \lambda\left(t-\delta T_{1}\right) \mathrm{d} t+\cdots \\
& +C_{f} \int_{T_{n-1}}^{T_{n}} \lambda\left(t-\delta T_{n-1}\right) \mathrm{d} t+C_{f} \int_{T_{n}}^{\frac{U_{0}}{r}} \lambda\left(t-\delta T_{n}\right) \mathrm{d} t+n C_{p} \\
= & C_{f} \sum_{i=1}^{n} \int_{T_{i-1}}^{T_{i}} \lambda\left(t-\delta T_{i-1}\right) \mathrm{d} t+C_{f} \int_{T_{n}}^{\frac{U_{0}}{r}} \lambda\left(t-\delta T_{n}\right) \mathrm{d} t+n C_{p}
\end{aligned}
$$

(4) When $r<\frac{U_{0}}{W_{0}}, n$ times of preventive maintenance are carried out and the warranty service is terminated at the end of the driving mileage. The cost is as follows:

$$
\begin{aligned}
E_{n+2}(C)= & C_{f} \int_{0}^{T_{1}} \lambda(t) \mathrm{d} t+C_{f} \int_{T_{1}}^{T_{2}} \lambda\left(t-\delta T_{1}\right) \mathrm{d} t+L+C_{f} \int_{T_{n-1}}^{T_{n}} \lambda\left(t-\delta T_{n-1}\right) \mathrm{d} t \\
& +C_{f} \int_{T_{n}}^{W_{0}} \lambda\left(t-\delta T_{n}\right) \mathrm{d} t+n C_{p} \\
= & C_{f} \sum_{i=1}^{n} \int_{T_{i-1}}^{T_{i}} \lambda\left(t-\delta T_{i-1}\right) \mathrm{d} t+C_{f} \int_{T_{n}}^{W_{0}} \lambda\left(t-\delta T_{n}\right) \mathrm{d} t+n C_{p}
\end{aligned}
$$

From Equations (1)-(4), we can get the total cost expectation of equipment warranty service during the warranty period as follows:

$$
\begin{aligned}
E(C) & =\int_{\frac{U_{0}}{T_{1}}}^{\infty} E_{1}(C) g(r) \mathrm{d} r+\int_{\frac{U_{0}}{T_{2}}}^{\frac{U_{0}}{T_{1}}} E_{2}(C) g(r) \mathrm{d} r+\cdots+\int_{\frac{U_{0}}{T_{n}}}^{\frac{U_{0}}{T_{n-1}}} E_{n}(C) g(r) \mathrm{d} r \\
& +\int_{\frac{U_{0}}{T_{n}}}^{\frac{U_{0}}{W_{0}}} E_{n+1}(C) g(r) \mathrm{d} r+\int_{0}^{\frac{U_{0}}{W_{0}}} E_{n+2}(C) g(r) \mathrm{d} r
\end{aligned}
$$

Finally, we can get the optimal model of warranty cost:

$$
\begin{cases}\min & E(C) \\ \text { s.t. } & T_{1} \in\left[T_{a 1}, T_{b 1}\right], T_{2} \in\left[T_{a 2}, T_{b 2}\right], \cdots, T_{n} \in\left[T_{a n}, T_{b n}\right]\end{cases}
$$




\section{Example Analysis}

It is known that the two-dimensional warranty period of a general equipment is three years or $150.000 \mathrm{~km}$ in the warranty contract. After consultation between the manufacturer and the army, only in the two periods from December of the first year to February of the second year and December of the second year to February of the third year can the manufacturer and the army carry out two preventive maintenances of the equipment. Preventive maintenance is incomplete maintenance. Minimum maintenance is used for equipment failure in the rest of the time. According to the statistical analysis of the historical data of the usage rate of the same type of equipment, the usage rate $r$ of the equipment obeys the Weibull distribution. The scale parameter is 1.2 , and the shape parameter is 4 . Other parameter settings are shown in Table 1 (relevant data can be obtained from field surveys conducted by engineering departments).

\begin{tabular}{|c|c|}
\multicolumn{2}{|c|}{ Table 1. Parameter settings } \\
\begin{tabular}{|c|c|}
\hline Parameter & Parameter values \\
\hline$\delta$ & 0.6 \\
\hline$C_{p} /$ yuan & 200 \\
\hline$C_{f} /$ yuan & 350 \\
\hline$\theta_{0}, \theta_{1}, \theta_{2}, \theta_{3}$ & $5 \times 10^{-7}, 10^{-7}, 2 \times 10^{-7}, 2 \times 10^{-7}$ \\
\hline
\end{tabular}
\end{tabular}

In order to simplify the calculation, in the three-year warranty period, the monthly calculation is based on 30 days, a total of 1080 days in three years. The time constraints of two preventive maintenances are [330, 420] and [690, 780].

Using MATLAB software and a numerical algorithm, $T_{1}$ takes value in $[330,420]$ and the step length takes five days, while $T_{2}$ takes value in [690, 780] and the step length is 5 days. We set up several sets of $\left(T_{1}, T_{2}\right)$ data. The corresponding costs of each group are calculated separately, and the results are shown in Figure 4. It can be seen that under different combinations of $T_{1}$ and $T_{2}$, the cost is different. The calculated cost surface has the lowest point, which indicates that there is the optimal $\left(T_{1}, T_{2}\right)$ to minimize the cost during the warranty period.

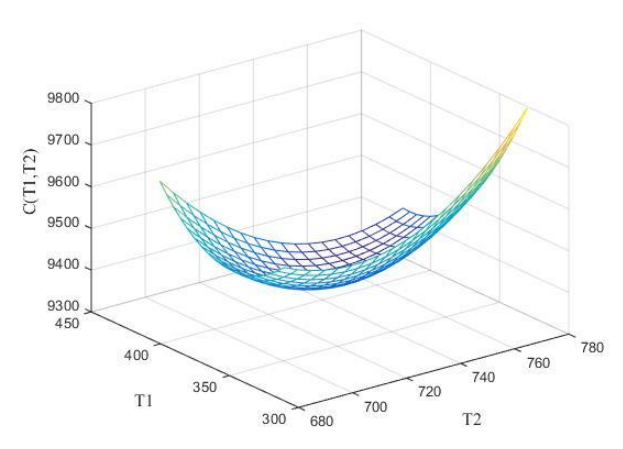

Figure 4. Warranty service cost

Through calculation, it is determined that the corresponding $T_{1}$ and $T_{2}$ of the minimum warranty cost are 395 and 745 respectively, and the corresponding warranty cost $E(C)$ is 9376 yuan. Select the best part of the cost data near the optimal $\left(T_{1}, T_{2}\right)$ to compare, as shown in Table 2.

Table 2. Costs for different preventive maintenance times

\begin{tabular}{|c|c|c|c|c|c|c|c|c|}
\hline \multicolumn{2}{|c|}{} & \multicolumn{7}{|c|}{$T_{1}$} \\
\cline { 3 - 10 } \multicolumn{2}{c|}{} & $\mathbf{3 8 0}$ & $\mathbf{3 8 5}$ & $\mathbf{3 9 0}$ & $\mathbf{3 9 5}$ & $\mathbf{4 0 0}$ & $\mathbf{4 0 5}$ & $\mathbf{4 1 0}$ \\
\hline \multirow{4}{*}{$T_{2}$} & $\mathbf{7 3 5}$ & 9379 & 9374 & 9372 & 9373 & 9377 & 9385 & 9395 \\
\cline { 2 - 10 } & $\mathbf{7 4 0}$ & 9378 & 9372 & 9369 & 9369 & 9371 & 9377 & 9386 \\
\cline { 2 - 10 } & $\mathbf{7 4 5}$ & 9380 & 9373 & 9368 & $\mathbf{9 3 6 7}$ & 9368 & 9372 & 9380 \\
\cline { 2 - 10 } & $\mathbf{7 5 0}$ & 9386 & 9376 & 9370 & 9367 & 9367 & 9370 & 9376 \\
\cline { 2 - 10 } & $\mathbf{7 5 5}$ & 939265 & 938244 & 93745 & 9370 & 9369 & 9370 & 9375 \\
\hline
\end{tabular}




\section{Result Analysis}

By changing different repair factors and time constraints, the corresponding changes in warranty costs are shown in Table 3.

\subsection{The Influence of the Change of Repair Factor on the Maintenance Cost}

By comparing schemes (1)-(4) in Table 3, it can be seen that under the condition of time constraints, the larger the repair factor, the smaller the cost of the whole warranty period, and the earlier the corresponding preventive maintenance time.

\subsection{The Influence of Time Constraints on Warranty Cost}

By comparing the schemes (3), (5), and (6) in Table 3, we can draw a conclusion. When the repair factor is fixed, the warranty cost may change with the change of time constraints. When the optimal preventive time is in the middle of the time constraint, the warranty cost is not affected by the time constraint boundary; when the optimal preventive time is equal to the time constraint boundary, the warranty cost is affected by the time constraint boundary. Moreover, the more the optimal preventive time takes the value of the time constraint boundary, the greater the impact, and the greater the warranty cost.

Table 3. Costs for different time constraints and repair factors

\begin{tabular}{|c|c|c|c|c|c|}
\hline Scheme & Time constraint & $\delta$ & Optimal $T_{1}$ & Optimal $T_{2}$ & Minimum cost/yuan \\
\hline$(1)$ & {$[330,420],[690,780]$} & 0.5 & 415 & 760 & 14484 \\
\hline$(2)$ & {$[330,420],[690,780]$} & 0.6 & 410 & 755 & 11736 \\
\hline$(3)$ & {$[330,420],[690,780]$} & 0.7 & 395 & 745 & 9367 \\
\hline$(4)$ & {$[330,420],[690,780]$} & 0.8 & 385 & 735 & 7368 \\
\hline$(5)$ & {$[410,500],[690,780]$} & 0.7 & 410 & 750 & 9373 \\
\hline$(6)$ & {$[410,500],[630,720]$} & 0.7 & 410 & 720 & 9432 \\
\hline$(7)$ & {$[310,400],[730,820]$} & 0.7 & 395 & 745 & 9367 \\
\hline$(8)$ & {$[410,500],[630,720]$} & 0.8 & 485 & 635 & 7368 \\
\hline
\end{tabular}

\subsection{Optimization Methods}

In order to eliminate the situation that the warranty cost is too high due to time constraints, two solutions are proposed. Firstly, without affecting the normal training of the army and the normal production of the manufacturer, the two sides negotiate to adjust the time constraints so as to optimize the maintenance cost. By comparing schemes (6) and (7) in Table 3, we can see that, secondly, when the constraint time cannot be changed, the repair factor can be changed. We can also see this when comparing schemes (6) and (8) in Table 3.

\section{Conclusions}

By introducing time constraints into the product warranty period, a model of product warranty cost considering time constraints is established for the first time in this paper. The optimal time for incomplete preventive maintenance within the constrained time is obtained through case study and cost considerations, which verifies the availability and effectiveness of the model.

Through the analysis of the results, the effects of time constraints and repair factors on the maintenance cost are given. This model can be used for reference to optimize the current large-scale equipment maintenance service cost, enhance the scientificity of the maintenance cost formulation during the warranty period, and have a strong reference value for manufacturers and users. This paper proposes a model on the premise that the cost of repairing maintenance is unchanged; however, in engineering practice, the cost of repairing maintenance within and outside time constraints may be different, which should be considered in the next step of research.

\section{Acknowledgements}

This research is supported by the National Natural Science Foundation of China and the Military Scientific Research Project.

\section{References}

1. M. Park and H. Pham, "A New Warranty Policy with Failure Times and Warranty Servicing Times," IEEE Transactions on Reliability, Vol. 61, No. 3, pp. 822-831, 2012 
2. R. S. Ramniwas, M. Kadyan, and J. Kumar, "Stochastic Modeling of a Single-Unit Repairable System with Preventive Maintenance under Warranty," International Journal of Computer Applications, Vol. 75, No. 14, pp. 36-41, 2014

3. C. Su and X. Wang, "A Two-Stage Preventive Maintenance Optimization Model Incorporating Two-Dimensional Extended Warranty," Reliability Engineering \& System Safety, Vol. 155, pp. 169-178, 2016

4. Y. S. Huang and C. Yen, "A Study of Two-Dimensional Warranty Policies with Preventive Maintenance," IIE Transactions, Vol. 41, No. 4, pp. 299-308, 2009

5. J. Wu, M. Xie, and T. S. Adam Ng, "On a General Periodic Preventive Maintenance Policy Incorporating Warranty Contracts and System Ageing Losses," International Journal of Production Economics, Vol. 129, No. 1, pp. 102-110, 2011

6. Y. S. Huang, W. Y. Gau, and J. W. Ho, "Cost Analysis of Two-Dimensional Warranty for Products with Periodic Preventive Maintenance," Reliability Engineering \& System Safety, Vol. 134, pp. 51-58, 2015

7. N. Tao and S. Zhang, "The Optimal Extended Warranty Length of Durable Goods based Preventive Maintenance Behavior," Systems Science \& Control Engineering, Vol. 3, No. 1, pp. 1-14, 2015

8. A. Y. Alqahtani and S. M. Gupta, "Money-Back Guarantee Warranty Policy with Preventive Maintenance Strategy for SensorEmbedded Remanufactured Products," Journal of Industrial Engineering International, Vol. 14, pp. 767-782, 2018

9. G. L. Liao, "Optimum Maintenance Strategy of a Repairable System under Long-Term Free Preventive Maintenance Warranty with Predicted Maintenance," International Journal of Reliability, Quality and Safety Engineering, Vol. 23, No, 4, pp. 17, 2016

10. J. Wang, Z. Zhou, and H. Peng, "Flexible Decision Models for a Two-Dimensional Warranty Policy with Periodic Preventive Maintenance," Reliability Engineering \& System Safety, Vol. 162, pp. 14-27, 2017

11. Y. Wang, Y. Liu, Z. Liu, and X. Li, "On Reliability Improvement Program for Second-Hand Products Sold with a TwoDimensional Warranty,” Reliability Engineering \& System Safety, Vol. 167, pp. 452-463, 2017

12. M. Shafiee, M. Finkelstein, and M. J. Zuo, "Optimal Burn-in and Preventive Maintenance Warranty Strategies with TimeDependent Maintenance Costs," IIE Transactions, Vol. 45, No. 9, pp. 1024-1033, 2013

13. X. Zhou, Y. Li, L. Xi, and J. Lee, "Multi-Phase Preventive Maintenance Policy for Leased Equipment," International Journal of Production Research, Vol. 53, No. 15, pp. 4528-4537, 2014

14. Y. Wang, Z. Liu, and Y. Liu, "Optimal Preventive Maintenance Strategy for Repairable Items under Two-Dimensional Warranty," Reliability Engineering \& System Safety, Vol. 142, pp. 326-333, 2015

15. M. Park, K. M. Jung, and D. H. Park, "Optimal Maintenance Strategy under Renewable Warranty with Repair Time Threshold," Applied Mathematical Modelling, Vol. 43, pp. 498-508, 2017

16. A. Nasrum, U.S. Pasaribu, and H. Husniah, "Service Contract with Periodic Preventive Maintenance for a Dump Truck Sold with a Two-Dimensional Warranty," in Proceedings of AIP Conference, 2016

17. C. Y. Cheng, X. F. Zhao, and M. Chen, "A Failure-Rate-Reduction Periodic Preventive Maintenance Model with Delayed Initial Time in a Finite Time Period," Quality Technology \& Quantitative Management, Vol. 11, No. 3, pp. 245-254, 2014

18. Y. C. Han, X. S. Jia, Y. S. Bai, and Y. T. Wu, "Optimization Research on Two-Dimensional Preventive Maintenance Intervals," Journal of Donghua University, Vol. 33, No. 2, pp. 196-200, 2016

19. H. Husniah, U. S. Pasaribu, and B. P. Iskandar, "A Servicing Strategy Involving Preventive Maintenance and Imperfect Repair for Two Dimensional Warranties," in Proceedings of CIE43, 2013

20. Y. S. Huang, "Two-Dimensional Warranty with Reliability-Based Preventive Maintenance," IEEE Transactions on Reliability, Vol. 62, No. 4, pp. 898-907, 2013

21. Z. L. Lin, Y. S. Huang, and C. C. Fang, "Non-Periodic Preventive Maintenance with Reliability Thresholds for Complex Repairable Systems," Reliability Engineering \& System Safety, Vol. 136, pp. 145-156, 2015

22. W. Y. Yun and K. M. Kang, "Imperfect Repair Policies under Two-Dimensional Warranty," Proceedings of the Institution of Mechanical Engineers, Part O: Journal of Risk and Reliability, Vol. 221, No. 4, pp. 239-247, 2007

Qian Wang is a master's student at the Shijiazhuang Campus of Army Engineering University. His research interests include equipment support theory and technology.

Zhonghua Cheng is a professor and doctoral supervisor at the Shijiazhuang Campus of Army Engineering University. His research interests include equipment support theory and technology.

Zhiyong Li is a teacher at the Shijiazhuang Campus of Army Engineering University. His research interests include equipment support theory

Yongsheng Bai is a teacher at the Shijiazhuang Campus of Army Engineering University. His research interests include maintenance engineering. 